\title{
WATER HYDRAULIC SYSTEM FOR HIGH SPEED CYLINDER DRIVE
}

\author{
Shigeru IKEO*, Hirotaka NAKASHIMA** and Kazuhisa ITO*** \\ * Faculty of Science and Engineering, Sophia University \\ 7-1 Kioicho, Chiyoda-ku, Tokyo, 102-8554 Japan \\ (E-mail: s_ikeo@sophia.ac.jp) \\ ** Future Architect Co. Ltd \\ *** Faculty of Engineering, Tottori University
}

\begin{abstract}
In order to apply the water hydraulic drive system to the precision die casting machine, the system with accumulator and logic valves are examined. As the pressure medium, water is environmental friendly, but it has disadvantage, e.g. leakage due to low viscosity, low lubricity and so on.

In this report, the friction force acting on logic valves were taken into account in the mathematical model, because for the purpose of prevention of leakage on logic valve two or three O-ring seals were used instead of labyrinth seal used in oil hydraulic logic valve. The validity of the mathematical model is assured by experimental results. Afterward the model is introduced into the mathematical model of whole high speed cylinder drive system and characteristics of the system is examined.
\end{abstract}

\section{KEY WORDS}

Key words; Water Hydraulics, High Speed Drive, Logic Valve, Mathematical Model, Friction Force

\section{INTRODUCTION}

Water hydraulics is a new power source having very high environmental friendliness that cannot achieved by conventional hydraulic systems while it has some technical problems to be solved. Japan Fluid Power System Society (JFPS) and Japan Fluid Power Association (JFPA) were and still are promoting its efficient application and supporting concerning projects. These several years are devoted to reveal the performance and the problems of conventional water hydraulic devices with practical experiments. In this project, the aim was 1) to know how high cylinder speed we can achieve, and 2) to clarify the obstacles which must to be solved in future.

In references [1], [2], we examined how to apply the water hydraulic technique for the injection and the dwelling processes of the hydraulic die casting machine. It was shown that the cylinder velocity is enhanced by three fold to seven fold by using the circuit combining the proper accumulator as auxiliary power source and logic valve than the case for using only directional control valve. Also we showed that the feasible highest cylinder velocity at medium supply pressure range up to $14 \mathrm{MPa}$ was $4.5 \mathrm{~m} / \mathrm{s}$ and at the water hydraulic cylinder stroke end the pressure rise was observed[3].

In this paper, our objectives are: 1) to establish the mathematical model of the water hydraulic system using 
logic valves, 2) to investigate pressure response in circuit and 3) to estimate pressure rise which occurs at the stoppage of the cylinder by simulation.

\section{SWITCHING CHARACTERISTICS OF LOGIC VALVE}

\section{Mathematical model}

In this research two types of logic valve, as shown in Figure 1, are used. Figure 2 shows the experimental circuit for testing the switching characteristics of logic valve.

The mathematical model of water hydraulic logic valve. which consists of continuity equations, equation of motion and equations on the relation between flow rate and pressure drop across the orifice, is similar to one for oil hydraulic logic valve [4]. Only the model of friction force is deferent, because for the purpose of prevention of leakage in water hydraulic logic valve, two or three O-ring seals were used instead of labyrinth seal used in oil hydraulic logic valve. The friction model is given as follows;

$$
\begin{aligned}
& F_{R N}=F_{R N 1}(x)-F_{R N_{2}}(\stackrel{\square}{x}) \\
& F_{R N 1}=\frac{x}{|x|+\mu} F_{s} \\
& F_{R N 2}=\left\{\begin{array}{l}
0 \quad \text { for } \quad|x| \leq V_{f r i} \\
\frac{x}{|\square|+\mu}\left(F_{s}-F_{d}\right) \quad \text { for } \quad|x| \geq V_{f r i} \text { : }
\end{array}\right.
\end{aligned}
$$

where $F_{R N 1}$ is the static friction term, $F_{R N 2}$ the dynamic friction term, $F s$ the maximum static friction force, $F d$ the dynamic friction force and $\mu$ the connecting factor. In water hydraulic logic valve, O-ring seals are used to prevent the leakage through the clearance between the valve body and the bush. Deformation of $\mathrm{O}$-ring is influenced by pressures acting both sides of O-ring. During the opening operation, the pressure of one side of O-ring is high, while the other side pressure is tank pressure. On the other hand, during the closing operation pressures on both sides are high. In order to take into the consideration of this situation, different values of $F s$ and $F d$ are adopted as shown in Table 1.

\section{Results of simulation and experiment}

As a example of experimental result, the displacement of valve body and pressures are shown in Figure 3 for Form A valve with fixed orifice $R x=R t=1.5 \mathrm{~mm}$ and load pressure $13 \mathrm{MPa}$. From experimental results conducted under changing the valve shape, load

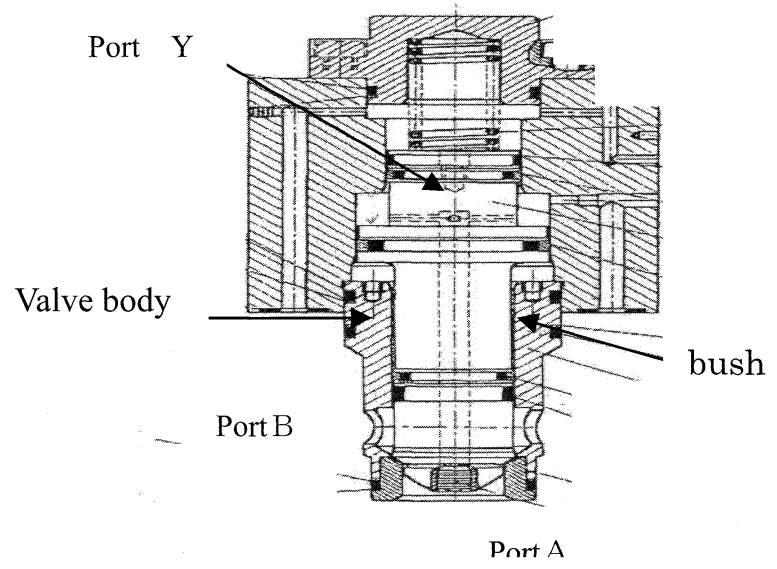

(a) Form A

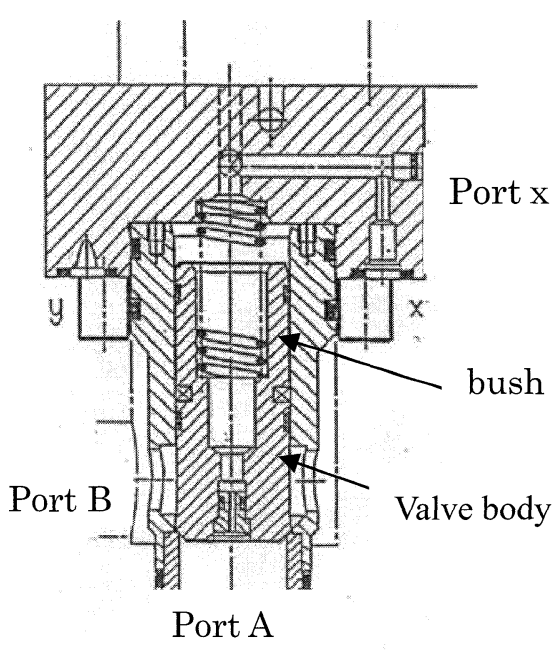

(b) Form A

Figure 1 Water hydraulic logic valve

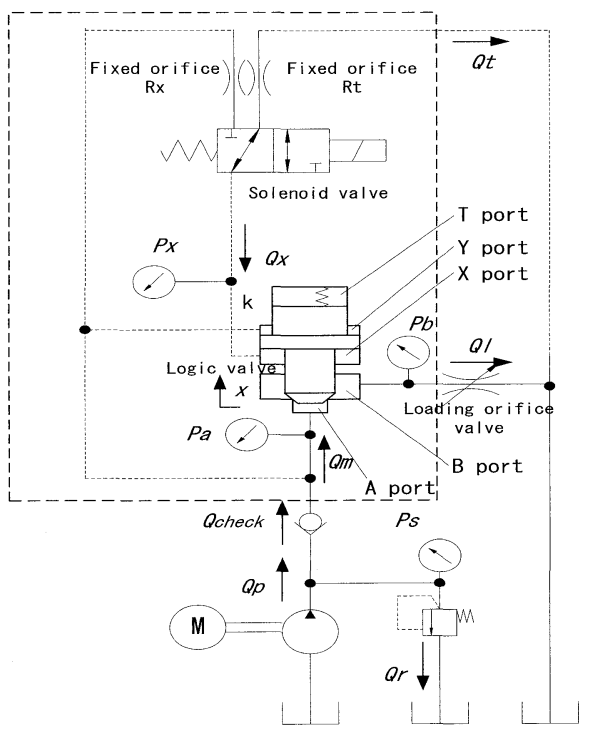

Figure 2 Experimental circuit 
Table 1 Friction force vs. pressure

\begin{tabular}{|c|c|c|c|c|}
\hline \multirow{2}{*}{$\begin{array}{c}\text { Pressure } \\
(\mathrm{MPa})\end{array}$} & \multicolumn{2}{|c|}{$\begin{array}{c}\text { Friction force } \\
\text { (valve open) }\end{array}$} & \multicolumn{2}{c|}{$\begin{array}{c}\text { Friction force (N) } \\
\text { (valve close) }\end{array}$} \\
\cline { 2 - 5 } & $\mathrm{F}_{\mathrm{s} 1}$ & $\mathrm{~F}_{\mathrm{d} 1}$ & $\mathrm{~F}_{\mathrm{s} 2}$ & $\mathrm{~F}_{\mathrm{d} 2}$ \\
\hline 13 & 2600 & 1400 & 210 & 150 \\
\hline 9 & 2200 & 1200 & 207 & 140 \\
\hline 4 & 2000 & 800 & 207 & 130 \\
\hline
\end{tabular}

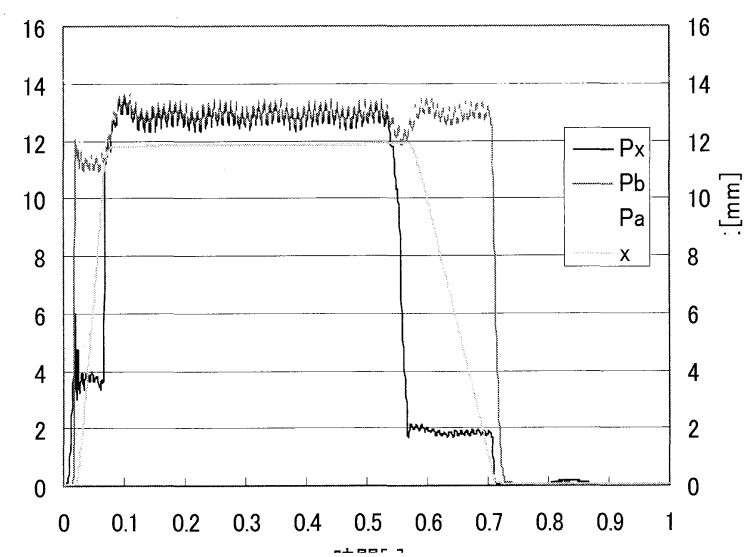

Figure 3 Experimental result

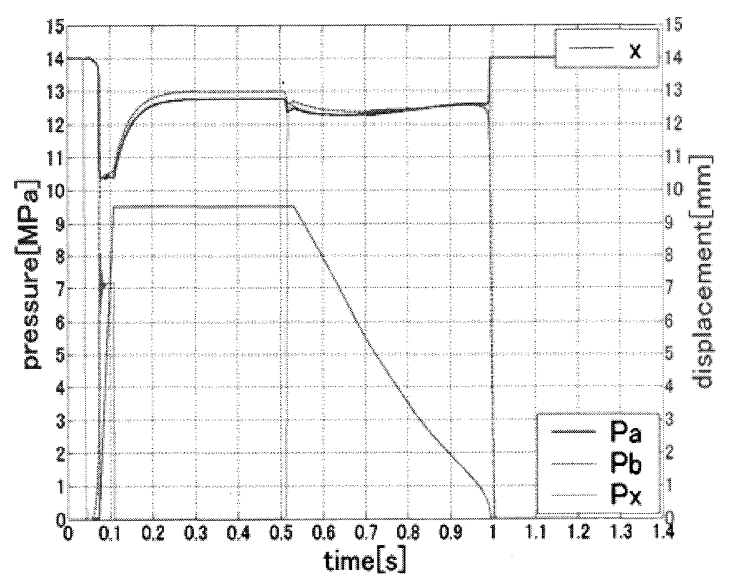

Figure 4 Resultsof simulation

2) the increase of load pressure make the closing time pressure and flow direction, it is seen that 1) the increase of load pressure make the opening time shorter, shorter in Form A valve and longer in Form B valve, 3) the opening and closing times for flow direction from A-port to B-port are shorter than ones for flow direction from B-port to A-port.

Figure 4 shows the result of simulation for Form $\mathrm{B}$ valve with fixed orifice $R x=R t=1.5 \mathrm{~mm}$ and load pressure 13MPa. Figure 5 shows the comparison of the experimental and simulated displacements.

From Figure 4, it is seen that the opening and closing

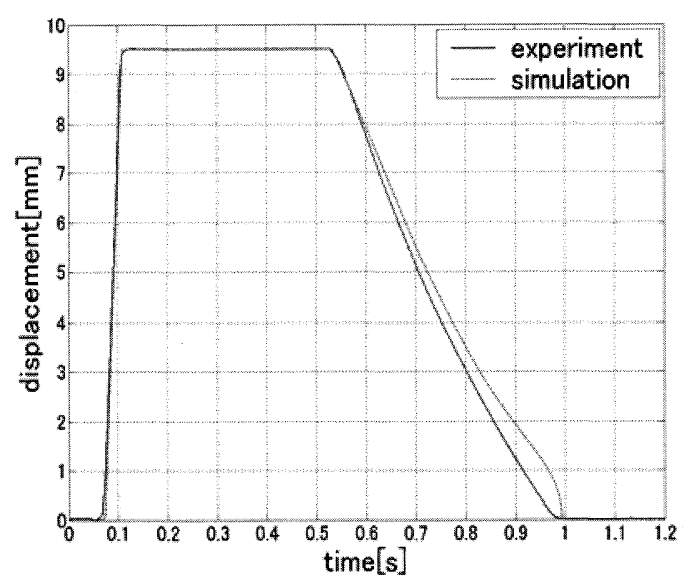

Figure 5 Comparison of experimental and simulated results

times given from simulation coincide with experimental results well. Experimental results on pressure variations are not shown here, they coincide well with results of simulation shown in Figure 4.

From these results the validity of the mathematical model is confirmed. Then, the dynamic characteristics of the water hydraulic system for high speed cylinder drive will be discussed in the next section.

\section{CHARACTERISTICS OF WATER HYDRAULIC HIGH SPEED CYLINDER DRIVE SYSTEM}

\section{Experimental setup}

Experimental apparatus and circuit are shown in Figure 6. This system is divided into three parts: the water supply unit, the water hydraulic cylinder and the load simulator unit. In the water supply unit, a fixed displacement axial piston pump is driven by the induction motor and $43.5 \mathrm{~L} / \mathrm{min}$ of flow is supplied at $1540 \mathrm{rpm}$. The pressure rating of this circuit is $14 \mathrm{MPa}$.

The high speed cylinder control circuit consisting of the accumulator and two logic valves is connected to the pump. The rod of the water hydraulic cylinder is connected to the same size oil hydraulic cylinder, which simulates the injection and the dwelling processes by loading the hydraulic cylinder. The load simulator is constructed with hydraulic cylinder and unload valve, and the PC controls the load/unload signal of this unit. The experimental parameters are as follows(see Fig.6):

- discharge pressure of pump(no.3) are set by the relief valve(no.10)

- load pressure (no.29) by the relief valve (no.31)

-pilot flow of logic valve (no.15) by changing the orifice (no.14) in pilot valve (no.12)

The following items are measured :

- rod and head pressure of water hydraulic cylinder (P28C, P28D)

—cylinder displacement $x$ 


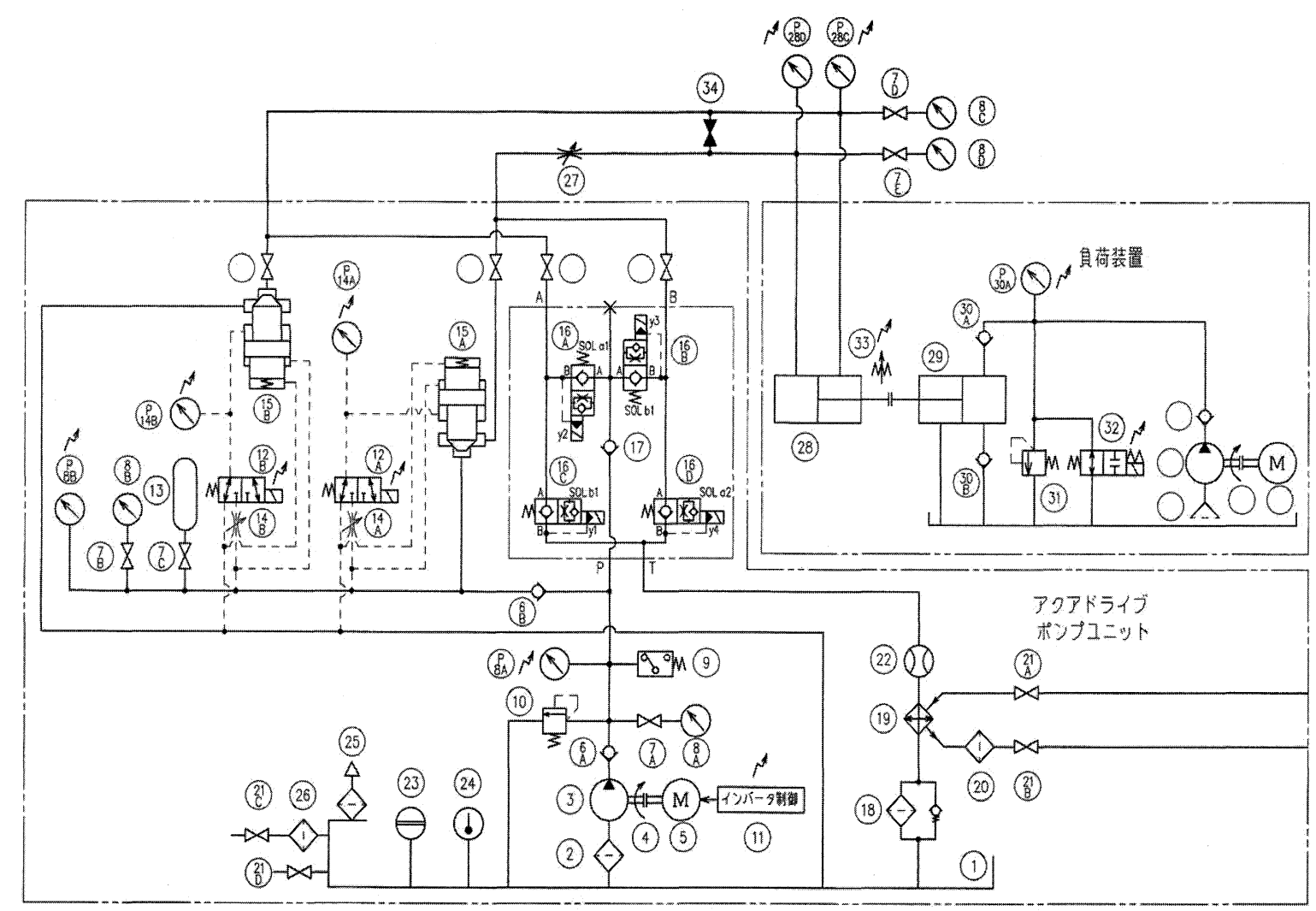

Figure 6 Water hydraulic high speed drive system

In this system, pressures acting two valves, $15 \mathrm{~A}$ and $15 \mathrm{~B}$. For the logic valve $15 \mathrm{~A}$ which delivers high pressure water to the cylinder head side port, high pressure in accumulator act at the port $\mathrm{A}$ and the load pressure in cylinder head side chamber at the port B. For the logic valve $15 \mathrm{~B}$ which delivers the water from the cylinder rod side port to tank, the pressure in cylinder rod side chamber act at the port $\mathrm{A}$ and the tank pressure at the port B. Since B-port pressure (load pressure) of valve $15 \mathrm{~B}$ is lower than the load pressure of valve $15 \mathrm{~A}$, valve $15 \mathrm{~B}$ start moving later than valve $15 \mathrm{~A}$ as explained before. Therefore pressure surge occurs in the cylinder head chamber. To avoid the pressure surge, it is necessary to operate the pilot valve $14 \mathrm{~B}$, which drives the logic valve $15 \mathrm{~B}$, some interval earlier than the pilot valve $14 \mathrm{~A}$, which drive the logic valve $15 \mathrm{~A}$.

In order to decide the proper time interval in the design stage, the mathematical model of the logic valve discussed before is used for the simulation of dynamic characteristic of whole water hydraulic high speed cylinder drive system

The rod of the water hydraulic cylinder is connected to the same size oil hydraulic cylinder, which simulates the injection and the dwelling processes by loading the hydraulic cylinder. The load simulator is constructed with hydraulic cylinder and unload valve, and the PC controls the load/unload signal of this unit.

\section{Experimental results}

Figure 7 shows the cylinder displacement and the pressure responses for $2.0 \mathrm{~mm}$ orifice diameter and $14 \mathrm{MPa}$ supply pressure and Figure 8 its close-up.

From Fig.6, the cylinder velocity decreases and the head pressure in the cylinder increases rapidly at the end of cylinder stroke. This is the surge pressure. Another pressure rise can be also observed at the rod/head of the cylinder when the rod starts to move. This comes from the time delay between two logic valves. This is due to the difference of the forces acting both valve bodies. Before opening the logic valves, logic valve (no.15A) is pressured by the supply pressure while the other (no.15B) by the return pressure from the water hydraulic cylinder. The former is much higher than the latter. Therefore the pressure in cylinder head rises as the logic valve (no.15A) opens because the logic valve (no.15B) is still closed. To suppress this pressure rise, we opened the pilot solenoid valve (no.12B) earlier than the no.12A so that this time lag would be decrease 


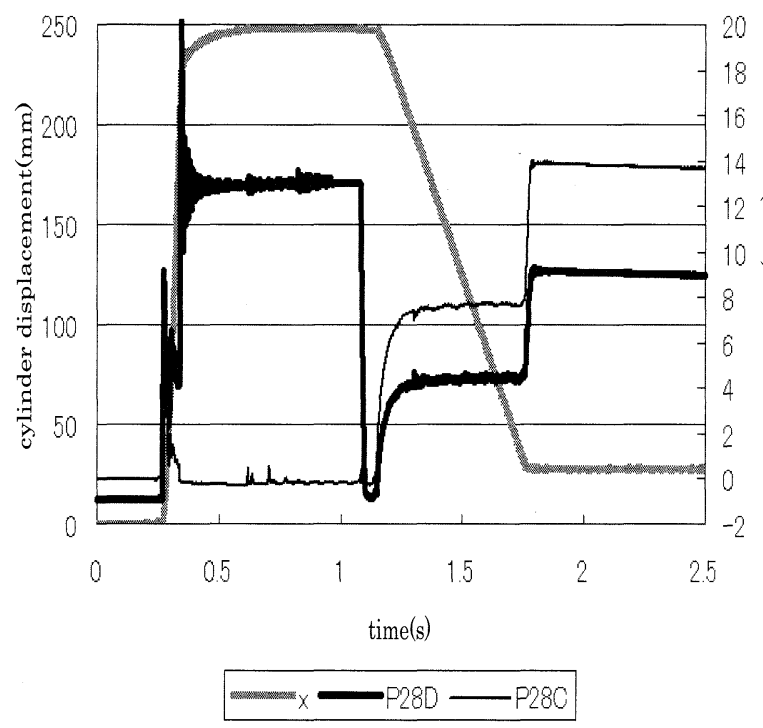

Figure 7 Cylinder displacement and pressure behavior

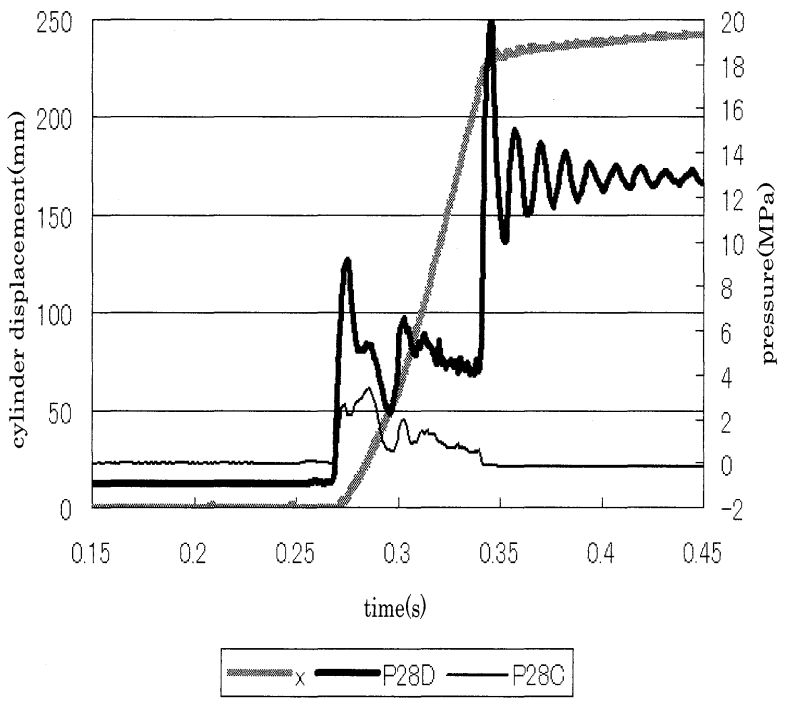

Figure 8 Cylinder displacement and pressure behavior (close-up)

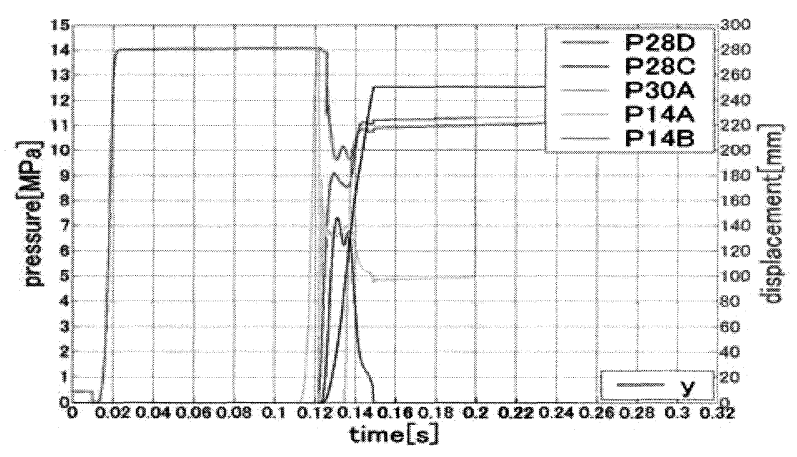

Figure 10 Results of simulation

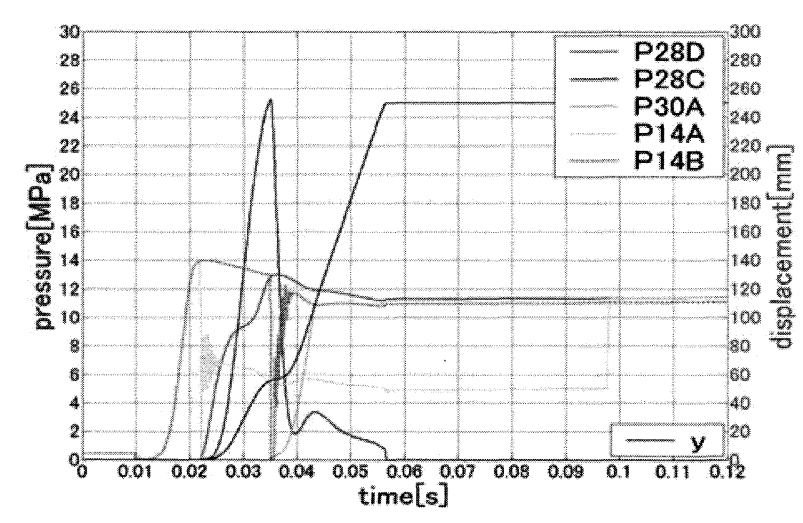

(a) displacement and pressures

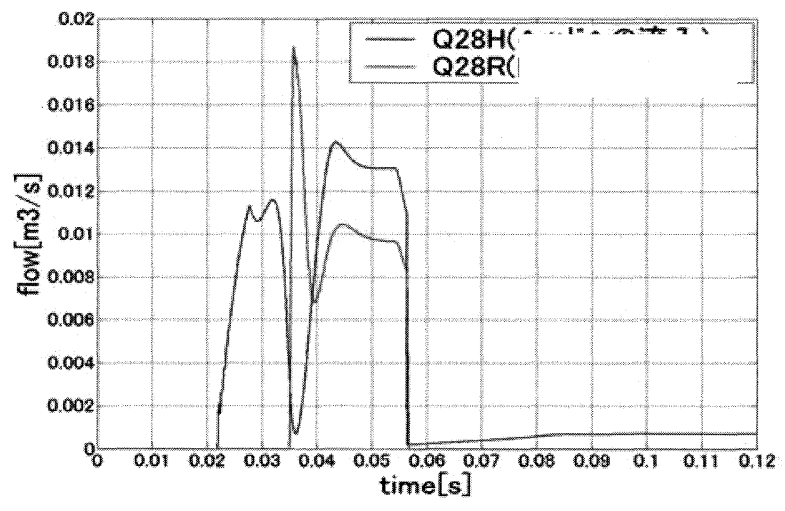

(b) Flow rates

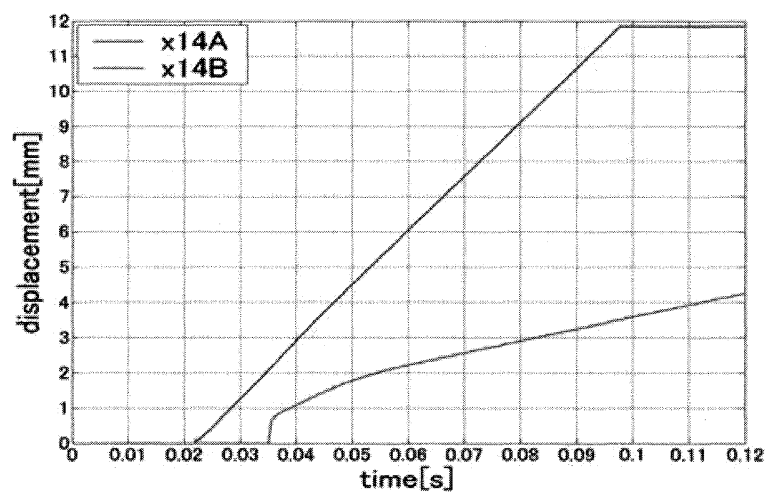

(c) Displacements of logic valves

Figure 9 Results of simulation

\section{Discussion}

Figure 9 shows the result of simulation when both pilot valves, 14A and 14B, operate simultaneously. Figure 9a shows pressures in cylinder head side chamber and rod side chamber P28D and P28C, pilot pressures P14A and $\mathrm{P} 14 \mathrm{~B}$, load simulator pressure $\mathrm{P} 30 \mathrm{~A}$ and cylinder displacement y. Figure $9 \mathrm{~b}$ shows flow rates into cylinder head chamber Q28A and out of cylinder rod chamber. Figure $9 \mathrm{c}$ shows displacements of both logic valve $\mathrm{x} 14 \mathrm{~A}$ and $\mathrm{x} 14 \mathrm{~B}$. 


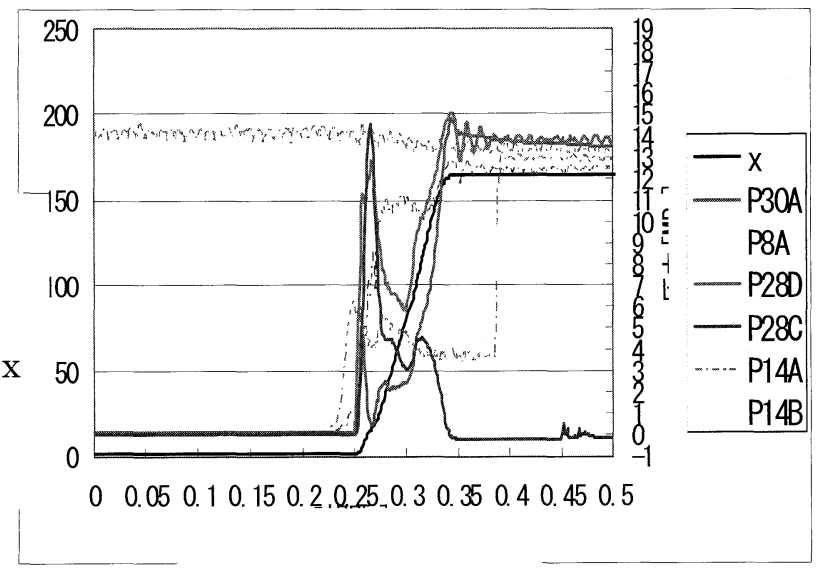

(a) without delay

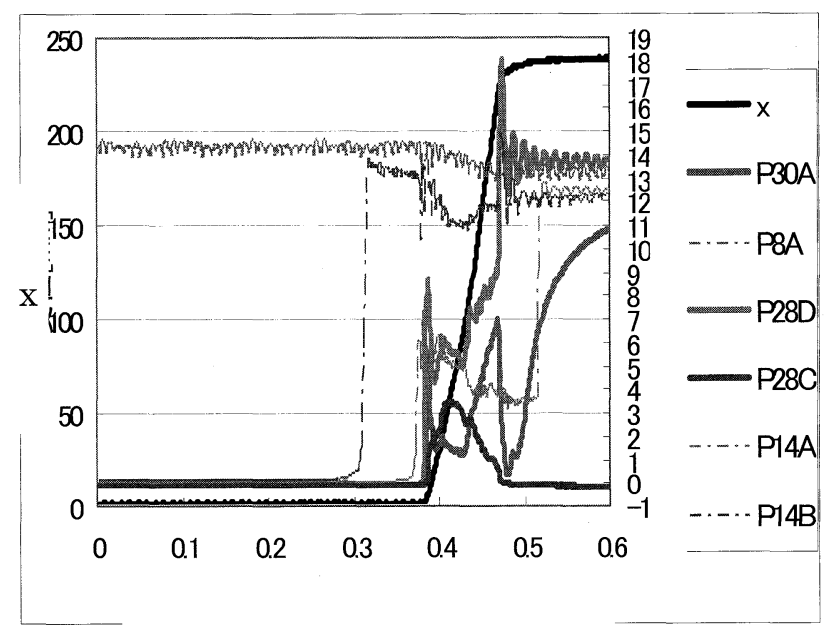

(b) with delay

Figure 11 Experimental Results

\section{Discussion}

Figure 9 shows the result of simulation when both pilot valves, $14 \mathrm{~A}$ and $14 \mathrm{~B}$, operate simultaneously. Figure 9a shows pressures in cylinder head side chamber and rod side chamber P28D and P28C, pilot pressures P14A and $\mathrm{P} 14 \mathrm{~B}$, load simulator pressure P30A and cylinder displacement $y$. Figure $9 \mathrm{~b}$ shows flow rates into cylinder head chamber Q28A and out of cylinder rod chamber. Figure 9c shows displacements of both logic valve $\mathrm{x} 14 \mathrm{~A}$ and $\mathrm{x} 14 \mathrm{~B}$.

Figure 10 shows the pressures and displacement of the logic valve from the simulation when the pilot valve $14 \mathrm{~A}$ operates $35 \mathrm{~ms}$ later than the pilot valve $14 \mathrm{~B}$. It is seen from this figure that the pressure surge can be depressed by operating the pilot valve $14 \mathrm{~A}$ later than pilot valve 14B. The time interval $35 \mathrm{~ms}$ was decided from simulation by trial and error.

Figure 11a shows the experimental results when both pilot valves are operated simultaneously and Figure $11 \mathrm{~b}$ when the pilot valve $14 \mathrm{~A}$ operates $50 \mathrm{~ms}$ later than the pilot valve 14B. From these results the effectiveness of operating time interval of both valves are confirmed.

\section{CONCLUSION}

In order to apply the water hydraulic drive system to the precision die casting machine, the system with accumulator and logic valves are examined. As the pressure medium, water is environmental friendly, but it has disadvantage, e.g. leakage due to low viscosity, low lubricity and so on.

(1) Mathematical model of water hydraulic logic valve is derived and its validity is assured experimentally. In the model, the friction force acting on logic valves were taken into account, because for the purpose of prevention of leakage on logic valve two or three O-ring seals were used instead of labyrinth seal used in oil hydraulic logic valve.

(2) The model is introduced into the mathematical model of whole high speed cylinder drive system and characteristics of the system is examined.

(3) In order to prevent the pressure surge which occurs when the cylinder rod starts to move, the method of operating the pilot valve $14 \mathrm{~A}$ later than $14 \mathrm{~B}$ is effective.

\section{Acknowledgement}

This research is supported by the committee "Practical Application of Acua Drive System Technique" of JFPA. Also we appreciate for Makome Research Institute for their support on the measurement of logic valve displacement.

\section{References}

1. Japan Fluid Power Association: Annual Survey Research Report on Practical Application of Aqua Drive System Technique 2003, 2004(in Japanese)

2. Japan Fluid Power Association: Aqua Drive System -A Technical Guide II, 2002

3. Shigeru Ikeo, Kazuhisa Ito and Takashi Ochiai: Research on High Speed Drive of Water Hydraulic Cylinder, Proceedings of $6^{\text {th }}$ International Conference on Fluid Power Transmission and Control, pp.208-211, Hangzhou, China, 2005.

4. Shigeru Ikeo, Koji Takahashi, Ryuji Miura and kunio Kanda: Switching Characteristics of Two-Way Cartridge Valve, Proceedings of $7^{\text {th }}$ International Fluid Power Symposium, pp.245-252, Bath, UK, 1986 